\title{
On the $C^{\infty}$-well-posedness of Goursat Problems
}

Dedicated to Professor S. Mizohata on the occasion of his 60th birthday

$$
\text { By }
$$

Takeshi MANDAI*

\section{$\S 0$. Introduction}

Many authors have investigated about null-solutions of characteristic Cauchy problems. When the coefficients are real-analytic, many systematic results have been obtained. When the coefficients are only $C^{\infty}$, however, few results are known. In this paper, as one of the cases when we can get well-parametrized null-solutions, we consider Goursat problems on $\boldsymbol{R}^{n+1}(n \geqq 1)$.

To give more explanation, we introduce some notations as follows;

$$
\begin{aligned}
(t, x, y) & =\left(t, x, y_{1}, \ldots, y_{n-1}\right) \text { are variables in } \mathbb{R}^{n+1} \\
\partial_{t} & =\partial / \partial t, D_{t}=-i \partial_{t} \text { etc, } D_{y}^{\alpha}=D_{y_{1}}^{\alpha_{1}} \ldots D_{y_{n-1}}^{\alpha_{n-1}} \text { where } \\
\alpha & =\left(\alpha_{1}, \ldots, \alpha_{n-1}\right) \text { is a multi-index }
\end{aligned}
$$

for a polynomial $p(t, x, y ; \tau, \xi, \eta)$ of $(\tau, \xi, \eta)$ with $C^{\infty}\left(\boldsymbol{R}^{n+1}\right)$-coefficients, we denote the homogeneous part of degree $h$ by $p_{h}(t, x, y ; \tau, \xi, \eta)$.

For a differential operator $P=p\left(t, x, y ; D_{t}, D_{x}, D_{y}\right)$ of order $m$ and an integer $r$ such that $0<r<m$, consider the following Goursat problem:

$$
\left\{\begin{array}{l}
P u=f(t, x, y) \text { on } \Omega=[0, T] \times \mathbb{R}^{n} \quad(T>0), \\
\partial_{t}^{j} u_{\mid t=0}=g_{j}(x, y) \text { on } \boldsymbol{R}^{n} \quad(0 \leqq j<m-r), \\
\partial_{x}^{k} u_{\mid x=0}=h_{k}(t, y) \text { on } \Omega_{0}=[0, T] \times \mathbb{R}^{n-1} \quad(0 \leqq k<r),
\end{array}\right.
$$

where $f, g_{j}, h_{k}$ are given $C^{\infty}$-functions and satisfy the compatibility-

Communicated by S. Matsuura, March 20, 1985.

* Research Institute for Mathematical Sciences, Kyoto University, Kyoto 606, Japan.

Present Address : Department of Mathematics, Faculty of General Education, Gifu University, Yanagido, Gifu 501-11, Japan. 
condition:

(C)

$$
\partial_{x}^{k} g_{j}(0, y)=\partial_{t}^{j} h_{k}(0, y) \text { on } \mathbb{R}^{n-1}(0 \leqq j<m-r, 0 \leqq k<r) .
$$

For simplicity, we assume all the coefficients of $P$ belong to $\mathscr{B}^{\infty}\left(\mathbb{R}^{n+1}\right)$ $=\left\{f \in C^{\infty}\left(\mathbb{R}^{n+1}\right) ; \partial_{t}^{j} \partial_{x}^{k} \partial_{y}^{\alpha} f\right.$ is bounded for any $\left.(j, k, \alpha)\right\}$. In the case of constant coefficients, Y. Hasegawa [1], T. Nishitani [5] have investigated the $C^{\infty}$-well-posedness of Goursat problems. Following them, we assume that the operator $P$ has the following structure throughout this paper.

$$
\begin{aligned}
& P=\sum_{j=0}^{m-r} c_{j}\left(t, x, y ; D_{x}, D_{y}\right) D_{t}^{m-r-j}, \text { where ord. } c_{j} \leqq r+j \\
& (0 \leqq j \leqq m-r) \text { and } c_{0, r}(t, x, y ; 1,0)=1 .
\end{aligned}
$$

Under this assumption, T. Nishitani [5] found a necessary and sufficient condition for $C^{\infty}$-well-posedness in the case of constant coefficients. His condition is the following.

$$
\begin{aligned}
& \text { There exists a positive constant } \varepsilon \text { such that the polynomial } \\
& p(\tau, \xi, \eta) \text { is hyperbolic with respect to }(\tau, \xi, \eta)=(1, \delta, 0) \\
& f_{\text {or }} \text { every } \delta \text { with } 0<|\delta| \leqq \varepsilon \text { 。 }
\end{aligned}
$$

This condition implies the following two conditions.

(i) $p_{m}(\tau, \xi, \eta)$ is divisible by $c_{0, r}(\xi, \eta)$, that is, there exists a polynomial $q_{m-r}(\tau, \xi, \eta)$ such that $p_{m}(\tau, \xi, \eta)=c_{0, r}(\xi, \eta) q_{m-r}(\tau, \xi, \eta)$. Further, $c_{0, r}$ is hyperbolic with respect to $(\xi, \eta)=(1,0)$ and $q_{m-r}$ is hyperbolic with respect to $(\tau, \xi, \eta)=(1,0,0)$.

(ii) $p_{m-1}(\tau, \xi, \eta)-c_{0, r-1}(\xi, \eta) q_{m-r}(\tau, \xi, \eta)$ is divisible by $c_{0, r}(\xi, \eta)$.

Taking these results into account, we want to know what kind of conditions should be imposed in the case of variable coefficients.

\section{\&1. Statement of Results}

We set the following assumption.

$$
\left\{\begin{array}{l}
p_{m}(t, x, y ; \tau, \xi, \eta)=c_{0, r}(t, x, y ; \xi, \eta) q_{m-r}(t, x, y ; \tau, \xi, \eta) \text {, where } \\
\text { (i) the equation } q_{m-r}(t, x, y ; \tau, \xi, \eta)=0 \text { with respect to } \tau \text { has } \\
\text { only real distinct roots for any }(t, x, y ; \xi, \eta) \in \Omega \times\left(\mathbb{R}^{n} \backslash\{0\}\right) \text {, } \\
\text { (ii) either } n=1 \text { or the equation } c_{0, r}(t, x, y ; \xi, \eta)=0 \text { with re- } \\
\text { spect to } \xi \text { has only real distinct roots for any }(t, x, y ; \eta) \in \\
\Omega \times\left(\mathbb{R}^{n-1} \backslash\{0\}\right) .
\end{array}\right.
$$


Definition 1. 1. 1) Put $\Sigma_{2}^{\prime}=\left\{\left(t,{ }^{p} x, y ; \tau, \xi, \eta\right) \in \Omega \times\left(\mathbb{R}^{n+1} \backslash\{0\}\right) ; c_{0, r}\right.$ $\left.=q_{m-r}=0\right\}$.

2) For a positive number $M$, put

$$
\left\{\begin{array}{l}
\Gamma_{1}\left(t_{0}, x_{1}, y_{1}\right)=\left\{(t, x, y) \in \Omega ;\left(\left|x-x_{1}\right|^{2}+\left|y-y_{1}\right|^{2}\right)^{1 / 2} \leqq M\left(t_{0}-t\right)\right\}, \\
\Gamma_{2}\left(x_{0}, y_{0}\right)=\left\{\begin{array}{l}
\left.\left\{(x, y) \in \mathbb{R}^{n} ;\left|y-y_{0}\right| \leqq M\left(x_{0}-x\right), x \geqq 0\right\} \text { (if } x_{0} \geqq 0\right), \\
\left.\left\{(x, y) \in \mathbb{R}^{n} ;\left|y-y_{0}\right| \leqq M\left(x-x_{0}\right), x \leqq 0\right\} \text { (if } x_{0} \leqq 0\right),
\end{array}\right. \\
\Gamma\left(t_{0}, x_{0}, y_{0}\right)=U\left\{\Gamma_{1}\left(t_{0}, x_{1}, y_{1}\right) ;\left(x_{1}, y_{1}\right) \in \Gamma_{2}\left(x_{0}, y_{0}\right)\right\} .
\end{array}\right.
$$

Theorem 1. Assume (A-1) and

$$
\left\{\begin{array}{l}
\sigma_{m-1}\left(P-q_{m-r}\left(t, x, y ; D_{t}, D_{x}, D_{y}\right) \circ c_{0, r}\left(t, x, y ; D_{x}, D_{y}\right)\right)=0 \text { on } \\
\Sigma_{2}^{\prime} \text { where } \sigma_{m-1}(Q) \text { denotes the principal symbol of } Q \text { as an } \\
\text { operator of order } m-1 \text { and } \circ \text { denotes the composition of two } \\
\text { operators. }
\end{array}\right.
$$

Then, for any $f \in C^{\infty}(\Omega)$, any $g_{j} \in C^{\infty}\left(\mathbb{R}^{n}\right)(0 \leqq j<m-r)$ and any $h_{k} \in C^{\infty}\left(\Omega_{0}\right)$ $(0 \leqq k<r)$ with $(G)$, there exists a unique solution $u \in C^{\infty}(\Omega)$ of $\left(G . P_{0}\right)$. Further, there exists a positive constant $M$ such that for any $\left(t_{0}, x_{0}, y_{0}\right) \in \Omega$, the set $\Gamma\left(t_{0}, x_{0}, y_{0}\right)$ is a dependence domain of $\left(t_{0}, x_{0}, y_{0}\right)$, that is, if $f=0$ on $\Gamma\left(t_{0}, x_{0}, y_{0}\right), g_{j}=0$ on $\Gamma\left(t_{0}, x_{0}, y_{0}\right) \cap\{t=0\}(0 \leqq j<m-r)$ and $h_{k}=0$ on $\Gamma\left(t_{0}, x_{0}, y_{0}\right) \cap\{x=0\}(0 \leqq k<r)$, then $u=0$ on $\Gamma\left(t_{0}, x_{0}, y_{0}\right)$.

If the conclusion of the theorem is satisfied, we say that the Goursat problem is $C^{\infty}$-well-posed with good dependence domains. This conception make it easy to get necessary conditions, like the conception of the existence of a finite propagation speed in the non-characteristic Cauchy problems. (Cf. T. Nishitani [6].)

Remark 1.2. (i) If $r=1$, this theorem has been essentially proved by Y. Hasegawa ([1]).

(ii) If $\mathrm{n}=1$, then $\Sigma_{2}^{\prime}=\phi$, hence (A-2) is satisfied.

Theorem 2. Assume (A-1) and

(A-3) $\left\{\begin{array}{l}\left\{q_{m-r}, c_{0, r}\right\}=0 \text { on } \Sigma_{2}^{\prime} \text {, where }\{,\} \text { denotes the Poisson bracket with } \\ \text { respect to }(t, x, y ; \tau, \xi, \eta) .\end{array}\right.$ If the Goursat problem is $C^{\infty}$-well-posed with good dependence domains, then (A-2) is satisfied.

Remark 1.3. Under the condition (A-3), the condition (A-2) is 
equivalent to

$(\mathrm{A}-2)^{\prime}\left\{\begin{array}{l}p_{m-1}^{s}=0 \text { on } \Sigma_{2}^{\prime}, \text { where } p_{m-1}^{s} \text { denotes the subprincipal symbol } \\ \text { of } P \text {. }\end{array}\right.$

It is natural to ask what kind of conditions on lower order terms are necessary, if (A-3) is not satisfied. As to this question, the author believes that without any conditions on lower order terms, the Goursat problem is $C^{\infty}$-well-posed with good dependence domains, if $(\mathrm{A}-1)$ and the following $(\mathrm{A}-4)$ is satisfied.

$$
\left\{q_{m-r}, c_{0, r}\right\} \neq 0 \text { on } \Sigma_{2}^{\prime} \text {. }
$$

We can prove, however, only the following.

Theorem 3. Assume $m=n=2, \quad r=1$ and that (A-1) is satisfied. Further, assume (A-4) and

$$
q_{m-r}=q_{1}(t, x, y ; \tau, \xi, \eta) \text { is independent of } \xi .
$$

Then, the Goursat problem is $C^{\infty}$-well-posed with good dependence domains.

Example 1.4. Consider $P=\partial_{t} \partial_{x}-x \partial_{x} \partial_{y}+$ (lower order terms) on $\boldsymbol{R}^{3}$. The Goursat problem for $P$ is $C^{\infty}$-well-posed with good dependence domains for any lower order terms.

\section{§2. Proof of Theorem 1}

The idea of our proof is the same as that of Hasegawa's ([1]).

By the assumption of (A-1), the following Cauchy problem is $C^{\infty}$-well-posed with a finite propagation speed.

$$
\left\{\begin{array}{l}
c_{0}\left(0, x, y ; D_{x}, D_{y}\right) v=w(x, y) \text { on } \boldsymbol{R}^{n}, \\
\partial_{x}^{k} v_{\mid x=0}=v_{k}(y) \text { on } \boldsymbol{R}^{n-1}(0 \leqq k<r) .
\end{array}\right.
$$

Solving suitable equations of this type, we can determine $\partial_{t}^{j} u_{\mid t=0}$ $(j \geqq m-r)$ uniquely from (G. P.), hence we may assume $g_{j}(x, y)=0$ $(0 \leqq j<m-r)$ and $f, h_{k}(0 \leqq k<r)$ are flat at $t=0$, that is, all the derivatives vanish at $t=0$. In this case, the solution $u$ of (G. P.) is also flat at $t=0$.

Let $Q$ be an arbitrary differential operator whose principal symbol is $q_{m-r}$ and put $C_{0}\left(t_{0}\right)=c_{0}\left(t_{0}, x, y ; D_{x}, D_{y}\right)$. To avoid ambiguity, we fix 
some terminologies.

Definition 2.1. 1) The set $\Gamma \subset \Omega$ (resp. $\gamma \subset \boldsymbol{R}^{n}$ ) is called a uniqueness domain of the Cauchy problem for $Q$ (resp. $\left.C_{0}\left(t_{0}\right)\right)$, if $u \in C^{\infty}\left(\boldsymbol{R}^{n+1}\right)$ (resp. $v \in C^{\infty}\left(\boldsymbol{R}^{n}\right)$ ) satisfies $Q u=0$ on $\Gamma$ and $\partial_{t}^{j} u_{\mid t=0}=0$ on $\Gamma \cap\{t=0\}$ $(0 \leqq j<m-r) \quad\left(\operatorname{resp} . C_{0}\left(t_{0}\right) v=0\right.$ on $\gamma$ and $\partial_{x}^{k} v_{\mid x=0}=0$ on $\gamma \cap\{x=0\} \quad(0 \leqq$ $k<r)$ ), then $u=0$ on $\Gamma$ (resp. $v=0$ on $\gamma$ ).

2) A dependence domain of $\left(t_{0}, x_{0}, y_{0}\right) \quad\left(\operatorname{resp} .\left(x_{0}, y_{0}\right)\right)$ of the Cauchy problem for $Q$ (resp. $C_{0}\left(t_{0}\right)$ ) is a uniqueness domain including $\left(t_{0}, x_{0}, y_{0}\right) \quad\left(\operatorname{resp} .\left(x_{0}, y_{0}\right)\right)$.

Let $M$ and $T_{0}$ be positive numbers such that $T_{0} \geqq 2 T$. Put $\gamma_{t}=\left\{(x, y) \in \boldsymbol{R}^{n} ;|y| \leqq M\left(T_{0}-t\right)\left(2 M T_{0}-|x|\right),|x| \leqq M\left(T_{0}-t\right)\right\}$ for $t \in$ $[0, T]$. The following lemma is easy, hence the proof is omitted.

Lemma 2.2. For sufficiently large $M$, the set $\gamma_{t_{0}}$ (resp. $\Gamma_{t_{0}}=\bigcup_{0 \leqq t \leqq t_{0}}\{t\}$ $\left.\times \gamma_{t}\right)$ is a uniqueness domain of the Ciauchy problem for $C_{0}\left(t_{0}\right)$ (resp. Q) for any $t_{0} \in[0, T]$.

The following energy inequalities are the main points of the proof. (See Lemma 6.1 and 6.2 in [1].)

Lemma 2.3. For any non-negative integers $p, q, s$, there exists a constant $C$ such that the following two inequalities hold.

$$
\begin{aligned}
& \sum_{j=0}^{p+m-r-1}\left\|\partial_{t}^{j} v(t, \cdot, \cdot)\right\| \|_{s+m-r-1-j, t} \\
& \leqq C \sum_{j=0}^{p} \int_{0}^{t}\left\|\partial_{t}^{j} Q v\left(t^{\prime}, \cdot, \cdot\right)\right\| \|_{s-j, t} d t^{\prime} \quad(0 \leqq t \leqq T),
\end{aligned}
$$

for any $v \in C_{+}^{\infty}\left(\boldsymbol{R}^{n+1}\right)=\left\{v \in C_{0}^{\infty}\left(\boldsymbol{R}^{n+1}\right) ; v=0\right.$ on $\left.t<0\right\}$.

$$
\begin{aligned}
& \sum_{j=0}^{q} \sum_{k=0}^{p+r-1-j}\left\|\partial_{t}^{j} \partial_{x}^{k} u(t, x, \cdot)\right\|_{p+r-1-k-j, t, x} \\
\leqq & C\left\{\sum_{j=0}^{q}\left\|\partial_{t}^{j} C_{0}(t) u(t, \cdot, \cdot)\right\| \|_{p-j, t}\right. \\
& \left.\quad+\sum_{j=0}^{q} \sum_{k=0}^{r-1}\left\|\partial_{t}^{j} \partial_{x}^{k} u(t, 0, \cdot)\right\|_{p+r-1-k-j, t, 0}\right\} \\
& \left(0 \leqq t \leqq T,|x| \leqq M\left(T_{0}-t\right)\right), \text { for any } u \in C_{+}^{\infty}\left(\mathbb{R}^{n+1}\right) .
\end{aligned}
$$

Here, $\||\cdot|\|_{s, t}$ denotes the Sobolev norm of order $s$ on the domain $\gamma_{t}$ and 
$\|\cdot\|_{s_{1, t}, x_{1}}$ denotes the Sobolev norm of order $s$ on the domain $\gamma_{t} \cap\left\{x=x_{1}\right\}$.

Proof. Let $\|\cdot\| \|_{s}$ (resp. $\|\cdot\|_{s}$ ) denote the Sobolev norm of order $s$ on $\boldsymbol{R}_{(x, y)}^{n}$ (resp. $\boldsymbol{R}_{y}^{n-1}$ ).

(1) It is well-known that there holds the following energy inequality for $Q$ on an arbitrary compact set $K$.

$$
\begin{aligned}
& \quad \sum_{j=0}^{p+m-r-1}\left\|\partial_{t}^{j} v(t, \cdot, \cdot)\right\| \|_{s+m-r-1-j} \\
& \quad \leqq C \sum_{j=0}^{p} \int_{0}^{t}\left\|\partial_{t}^{j} Q v\left(t^{\prime}, \cdot, \cdot\right)\right\| \|_{s-j} d t^{\prime} \quad(0 \leqq t \leqq T),
\end{aligned}
$$

for any $v \in C_{+}^{\infty}\left(\mathbb{R}^{n+1}\right)$ with supp $v \subset K$.

Since $\Gamma_{t}$ is a uniqueness domain of the Cauchy problem for $Q$, the inequality (2-1) follows from this.

(2) It is also well-known that there holds the following energy inequality for $C_{0}(t)$.

$$
\begin{gathered}
\sum_{j=0}^{q} \sum_{k=0}^{p+r-1-j}\left\|\partial_{t}^{j} \partial_{x}^{k} u(t, x, \cdot)\right\|_{p+r-1-k-j} \\
\leqq C \sum_{j=0}^{q}\left\{\sum_{k=0}^{p-j}\left|\int_{0}^{x}\left\|\partial_{t}^{j} \partial_{x}^{k} C_{0}(t) u\left(t, x^{\prime}, \cdot\right)\right\|_{p-k-j} d x^{\prime}\right|\right. \\
\left.\quad+\sum_{k=0}^{p+r-1-j}\left\|\partial_{t}^{j} \partial_{x}^{k} u(t, 0, \cdot)\right\|_{p+r-1-k-j}\right\} \\
\left(0 \leqq t \leqq T,|x| \leqq M T_{0}\right), \text { for any } u \in C_{0}^{\infty}(K) .
\end{gathered}
$$

If $x_{1} \geqq 0$ (resp. $x_{1} \leqq 0$ ), then $\gamma_{t} \cap\left\{0 \leqq x \leqq x_{1}\right\}$ (resp. $\gamma_{t} \cap\left\{x_{1} \leqq x \leqq 0\right\}$ ) is a uniqueness domain of the Cauchy problem for $C_{0}(t)$. Therefore, the following holds.

$$
\begin{gathered}
\sum_{j=0}^{q} \sum_{k=0}^{p+r-1-j}\left\|\partial_{t}^{j} \partial_{x}^{k} u(t, x, \cdot)\right\|_{p+r-1-k-j, t, x} \\
\leqq C \sum_{j=0}^{q}\left\{\sum_{k=0}^{p-j}\left|\int_{0}^{x}\left\|\partial_{t}^{j} \partial_{x}^{k} C_{0}(t) u\left(t, x^{\prime}, \cdot\right)\right\|_{p-k-j, t, x^{\prime}} d x^{\prime}\right|\right. \\
\left.+\sum_{k=0}^{p+r-1-j}\left\|\partial_{t}^{j} \partial_{x}^{k} u(t, 0, \cdot)\right\|_{p+r-1-k-j, t, 0}\right\}
\end{gathered}
$$

We can easily obtain

$$
\begin{aligned}
& \sum_{j=0}^{q} \sum_{k=0}^{p+r-1-j}\left\|\partial_{t}^{j} \partial_{x}^{k} u(t, 0, \cdot)\right\|_{p+r-1-k-j, t, 0} \\
& \leqq C \sum_{j=0}^{q}\left\{\sum_{i=0}^{p-1-j}\left\|\partial_{t}^{j} \partial_{x}^{l} C_{0}(t) u(t, 0, \cdot)\right\|_{p-1-l-j . t, 0}\right. \\
& \left.\quad+\sum_{k=0}^{r-1}\left\|\partial_{t}^{j} \partial_{x}^{k} u(t, 0, \cdot)\right\|_{p+r-1-k-j, t, 0}\right\}
\end{aligned}
$$




$$
\begin{aligned}
\leqq & C \sum_{j=0}^{q}\left\{\left\|\partial_{t}^{j} C_{0}(t) u(t, \cdot, \cdot)\right\|_{p-j, t}\right. \\
& \left.+\sum_{k=0}^{r-1}\left\|\partial_{t}^{j} \partial_{x}^{k} u(t, 0, \cdot)\right\|_{p+r-1-k-j, t, 0}\right\} .
\end{aligned}
$$

Further, we have

$$
\begin{aligned}
& \sum_{l=0}^{p-j}\left|\int_{0}^{x}\left\|\partial_{t}^{j} \partial_{x}^{l} C_{0}(t) u\left(t, x^{\prime}, \cdot\right)\right\|_{p-l-j, t, x^{\prime}} d x^{\prime}\right| \\
& \leqq G\left|\left\|\partial_{t}^{j} C_{0}(t) u(t, \cdot, \cdot)|\||_{p-j, t}, \text { if }|x| \leqq M\left(T_{0}-t\right) .\right.\right.
\end{aligned}
$$

Thus, we get (2-2).

Corollary 2.4. For any non-negative integers $p$ and $s$, there exists a constant $C$ such that the following inequality holds.

$$
\begin{aligned}
& \quad \sum_{j=0}^{p+m-r-1} \sum_{k=0}^{s+m-2-j}\left\|\partial_{t}^{j} \partial_{x}^{k} u(t, x, \cdot)\right\|_{s+m-2-j-k, t, x} \\
& \leqq C\left\{\sum_{j=0}^{p} \int_{0}^{t}\left\|\partial_{t}^{j} Q \circ C_{0}\left(t^{\prime}\right) u\left(t^{\prime}, \cdot, \cdot\right)\right\| \|_{s-j, t} d t^{\prime}\right. \\
& \left.\quad+\sum_{k=0}^{r-1} \sum_{j=0}^{p+m-r-1}\left\|\partial_{t}^{j} \partial_{x}^{k} u\left(t, 0,0^{\circ}\right)\right\|_{s+m-2-j-k, t, 0}\right\} \\
& \left(0 \leqq t \leqq T,|x| \leqq M\left(T_{0}-t\right)\right), \text { for any } u \in C_{+}^{\infty}\left(\mathbb{R}^{n+1}\right)
\end{aligned}
$$

Lemma 2.5. Assume (A-1) and (A-2). Then, the operator $P$ can be decomposed as follows.

$$
P=Q \circ C_{0}(t)+R \text {. }
$$

Here, $Q$ is a differential operator with the principal symbol $q_{m-r}$ and $R$ is a differential operator whose total order is less than $m-1$ and whose order with respect to $\partial_{t}$ is less than $m-r$.

Proof. Put $r(t, x, y ; \tau, \xi, \eta)=\sigma_{m-1}\left(P-q_{m-r}\left(t, x, y ; D_{t}, D_{x}, D_{y}\right) \circ\right.$ $\left.c_{0, r}\left(t, x, y ; D_{x}, D_{y}\right)\right)$. We can write

$$
\begin{aligned}
r(t, x, y ; \tau, \xi, \eta)= & c_{0, r-1}(t, x, y ; \xi, \eta) q_{m-r}(t, x, y ; \tau, \xi, \eta) \\
& +d(t, x, y ; \tau, \xi, \eta),
\end{aligned}
$$

where the degree of $d$ with respect to $\tau$ is less than $m-r$. By the assumption (A-2), we have $d=0$ on $\Sigma_{2}^{\prime}$. Let $\tau=\tau_{j}(t, x, y ; \xi, \eta)$ $(1 \leqq j \leqq m-r)$ be the roots of $q_{m-r}=0$. We have $d(t, x, y ; \tau ;(t, x, y$; $\xi, \eta), \xi, \eta)=0(1 \leqq j \leqq m-r)$ if $c_{0, r}(t, x, y ; \xi, \eta)=0$. Put $d(t, x, y$; $\tau, \xi, \eta)=\sum_{j=0}^{m-r-1} d_{j}(t, x, y ; \xi, \eta) \tau^{m-r-1-j} . \quad$ Since $\tau_{j}(1 \leqq j \leqq m-r)$ are 
distinct for $(\xi, \eta) \neq(0,0)$, we have $d_{j}(t, x, y ; \xi, \eta)=0 \quad(0 \leqq j<m-r)$ if $c_{0, r}(t, x, y ; \xi, \eta)=0$ and $(\xi, \eta) \neq(0,0)$. Since $c_{0, r}=0$ has only real distinct roots $\xi$ for $\eta \neq 0$, there follows that $d_{j}(0 \leqq j<m-r)$ are divisible by $c_{0, r}$. Put $d=c_{0, r} q_{m-r-1}$. Put $Q=\left(q_{m-r}+q_{m-r-1}\right) \quad(t, x, y$; $\left.D_{t}, D_{x}, D_{y}\right)$ and $R=P-Q \circ C_{0}(t)$. Then, the operator $R$ has the desired properties.

Now, we shall construct the solution of (G. P.). Solving Cauchy problems for $Q$ and $\mathrm{C}_{0}(t)$, we can determine $u_{l}(l \geqq 0)$ by the following iteration.

$$
\begin{aligned}
& \left\{\begin{array}{l}
Q \circ C_{0}(t) u_{0}=f(t, x, y) \text { on } \Omega, \\
\partial_{t}^{j}\left(C_{0}(t) u_{0}\right)_{\mid t=0}=0 \text { on } R^{n} \quad(0 \leqq j<m-r), \\
\partial_{x}^{k} u_{0 \mid x=0}=h_{k}(t, y) \text { on } \Omega_{0} \quad(0 \leqq k<r) .
\end{array}\right. \\
& \left\{\begin{array}{l}
Q \circ C_{0}(t) u_{l+1}=-R u_{l} \text { on } \Omega, \\
\partial_{t}^{j}\left(C_{0}(t) u_{l+1}\right)_{\mid t=0}=0 \text { on } R^{n} \quad(0 \leqq j<m-r), \\
\partial_{x}^{k} u_{l+1 \mid x=0}=0 \text { on } \Omega_{0} \quad(0 \leqq k<r), \quad(l \geqq 0) .
\end{array}\right.
\end{aligned}
$$

By Corollary 2. 4, there holds

$$
\begin{aligned}
& \sum_{j=0}^{p}\left\{\|\| \partial_{t}^{j} R u(t, \cdot, \cdot)\left|\left\|\left.\right|_{s-j, t}+\right\|\right| \partial_{t}^{j} u(t, \cdot, \cdot)\|\|_{s+m-2-j, t}\right\} \\
& \leqq C\left\{\sum_{j=0}^{p} \int_{0}^{t} \mid\left\|\partial_{t}^{j} Q \circ C_{0}\left(t^{\prime}\right) u\left(t^{\prime}, \cdot, \cdot\right)\right\| \|_{s-j, t} d t^{\prime}\right. \\
& \left.\quad+\sum_{k=0}^{r-1} \sum_{j=0}^{p+m-r-1}\left\|\partial_{t}^{j} \partial_{x}^{k} u(t, 0, \cdot)\right\|_{s+m-2-j-k, t, 0}\right\} .
\end{aligned}
$$

Therefore, the infinite series $\sum_{l=0}^{\infty} u_{l}$ converges in $C^{\infty}(\Omega)$ and gives a solution $u$ of (G. P.). The uniqueness of the solution also follows from (2-3). Since for sufficiently large $M$, the set $\Gamma_{1}\left(t_{0}, x_{0}, y_{0}\right)$ (resp. $\left.\Gamma_{2}\left(x_{0}, y_{0}\right)\right)$ is a dependence domain of $\left(t_{0}, x_{0}, y_{0}\right)\left(\operatorname{resp} .\left(x_{0}, y_{0}\right)\right)$ of the Cauchy problem for $Q$ (resp. $C_{0}\left(t_{0}\right)$ ), it follows from the construction of solutions that $\Gamma\left(t_{0}, x_{0}, y_{0}\right)$ is a dependence domain of $\left(t_{0}, x_{0}\right.$, $y_{0}$ ) of the Goursat problem for $P$.

\section{§3. Proof of Theorem 2}

For a positive constant $\delta$, consider the following coordinate transformation: $s=t \pm \delta x, z=x, w=y$. Let $P_{ \pm}$be the transformed operator from $P$, that is, $P_{ \pm}^{\sim}=p\left(s \mp \delta z, z, w ; D_{s}, D_{z} \pm \delta D_{s}, D_{w}\right)$. From the 
assumption that the Goursat problem is $C^{\infty}$-well-posed with good dependence domains, it follows that for any $\left(t_{0}, x_{0}, y_{0}\right) \in \Omega$, there exist a neighborhood $U$ of $\left(t_{0}, x_{0}, y_{0}\right)$ and constants $N, C$ such that

$$
\begin{aligned}
& \left|u\left(t_{1}, x_{1}, y_{1}\right)\right| \leqq C \sum_{j+k+|\alpha| \leqq N} \sup _{\Gamma\left(t_{1}, x_{1}, y_{1}\right)}\left|\partial_{t}^{j} \partial_{x}^{k} \partial_{y}^{\alpha} P u\right| \\
& \text { for any } u \in C_{0}^{\infty}(U) \text { and any }\left(t_{1}, x_{1}, y_{1}\right) \in U .
\end{aligned}
$$

From this inequality, there holds the following inequality for sufficiently small positive number $\delta$ : For any $\left(\mathrm{t}_{0}, x_{0}, y_{0}\right)$ with $\pm x_{0}>0$, there exist a neighborhood $U^{\sim}$ of $\left(s_{0}, z_{0}, w_{0}\right)=\left(t_{0} \pm \delta x_{0}, x_{0}, y_{0}\right)$ and constants $N$, $C, M^{\sim}$ such that

$$
\left|u^{\sim}\left(s_{1}, z_{1}, w_{1}\right)\right| \leqq C \sum_{j+k+|\alpha| \leqq N} \sup _{\Gamma^{\sim}\left(s_{1}, z_{1}, w_{1}\right)}\left|\partial_{s}^{j} \partial_{z}^{k} \partial_{w}^{\alpha} P_{ \pm}^{\sim} u^{\sim}\right|
$$

for any $u^{\sim} \in C_{0}^{\infty}\left(U^{\sim}\right)$ and any $\left(s_{1}, z_{1}, w_{1}\right) \in U^{\sim}$,

where $\Gamma^{\sim}\left(s_{1}, z_{1}, w_{1}\right)=\left\{(s, z, w) ;\left(\left|z-z_{1}\right|^{2}+\left|w-w_{1}\right|^{2}\right)^{1 / 2} \leqq M^{\sim}\left(s_{1}-s\right)\right\}$. Now, put $q_{\tilde{m}-r}(s, z, w ; \sigma, \zeta, \omega)=q_{m-r}(s \pm \delta z, z, w ; \sigma, \zeta \pm \delta \sigma, \omega)$ and $\tilde{c_{0, r}}(s, z, w ; \sigma, \zeta, \omega)=c_{0, r}(s \pm \delta z, z, w ; \zeta \pm \delta \sigma, \omega)$. Then, we have $\tilde{p_{ \pm}, m}(s, z, w ; \sigma, \zeta, \omega)=p_{m}(s \pm \delta z, z, w ; \sigma, \zeta \pm \delta \sigma, \omega)=q_{\tilde{m}-r}^{\tilde{n}}(s, z, w ; \sigma$, $\zeta, \omega) c_{0, r}(s, z, w ; \sigma, \zeta, \omega)$. By the assumption (A-3) and the invariance of the Poisson bracket with respect to coordinate transformations, we have $\left\{q_{\tilde{m}-r}^{\tilde{c}}, \tilde{c_{0, r}}\right\}^{\sim}=0$ on $\Sigma_{2}^{\prime}=\left\{(\mathrm{s}, z, w ; \sigma, \zeta, \omega) ; q_{m-r}^{\tilde{m}}=\right.$ $\left.\tilde{c_{0 . r}}=0\right\}$, where $\{,\}^{\sim}$ is the Poisson bracket with respect to $(s, z, w$; $\sigma, \zeta, \omega)$. Since we can apply Theorem 2 of Ivrii-Petkov [2], we have $p_{m-1}^{\tilde{s}}=0$ on $\Sigma_{2}^{\prime \sim}$, where $p_{m-1}^{\sim s}$ is the subprincipal symbol of $P^{\sim}$. By the invariance of the subprincipal symbol, we have (A-2)'. It is an easy calculation to show that $(\mathrm{A}-2)^{\prime}$ is equivalent to (A-2) under the assumption (A-3).

\section{§4. Proof of Theorem 3}

Assume that $n=m=2$ and $p_{2}=(\tau-a(t, x, y) \eta)(\xi-b(t, x, y) \eta)$, where $\{\tau-a \eta, \xi-b \eta\} \neq 0$ on $\Sigma_{2}^{\prime}=\left\{(t, x, y ; \tau, \xi, \eta) \in \Omega \times R^{3} ; \tau=a \eta\right.$, $\xi=b \eta, \eta \neq 0\}$. Note that $\Sigma_{2}^{\prime}$ is the critical set of $p_{2}$, that is, $\Sigma_{2}^{\prime}=$ $\left\{(t, x, y ; \tau, \xi, \eta) \in \Omega \times R^{3} ; p_{2}=0, \nabla_{(t, x, y ; \tau, \xi, \eta)} p_{2}=0, \eta \neq 0\right\} . \quad$ As shown in $\S 2$, we can reduce the Goursat problem to the case where $g_{0}(x, y)$ $=0$ and $f, h_{0}$ are flat at $t=0$. Similarly, by the assumption (A-5), we can further reduce to the case where $g_{0}=h_{0}=0$ and $f$ is flat on $\{t=0$ or $x=0\}$. Thus, we have only to solve the followihg reduced 
problem.

$(\text { R. G. P. })_{ \pm}$

$$
\left\{\begin{array}{l}
P u_{ \pm}=f_{ \pm}(t, x, y) \text { on } \Omega, \\
u_{ \pm \mid t=0}=0 \text { on } R^{2}, \\
u_{ \pm \mid x=0}=0 \text { on } \Omega_{0},
\end{array}\right.
$$

where $f_{ \pm} \in C^{\infty}\left(R^{3}\right)$ and $f_{ \pm}=0$ on $S_{ \pm}=\{(t, x, y) ; t \leqq 0$, or $\pm x \leqq 0\}$.

Consider the coordinate transformation; $s=t \pm x, z=x, w=y$. Let $P_{ \pm}^{\sim}$ be the transformed operator from $P$. The principal symbol $p_{ \pm, 2}^{\sim}$ $= \pm(\sigma-a \omega)(\sigma \pm \zeta \mp b \omega)$ is effectively hyperbolic on the critical set of $p_{ \pm, 2}^{\tilde{a}}$ Therefore, by Theorem 2 of V. Ya. Ivrii [3] (cf. N. Iwasaki [4]), we can solve the following Cauchy problem for $P_{ \pm}^{\sim}$;

$$
\text { (C. P.) })_{ \pm}\left\{\begin{array}{l}
P_{ \pm}^{\tilde{u}} \tilde{u_{ \pm}}=f_{ \pm}(s, z, w)=f_{ \pm}(s \mp z, z, w) \text { on } R^{3}, \\
u_{ \pm \mid s=0}=\partial_{s} u_{ \pm \mid s=0}=0 \text { on } R^{2} .
\end{array}\right.
$$

Note that $f_{ \pm}^{\tilde{ \pm}}=0$ on $S_{ \pm}^{\sim}=\{(s, z, w) ; s \mp z \leqq 0, \pm z \leqq 0\}$. By the wellknown sweep-out method, we can prove that

$$
\begin{aligned}
\Gamma_{ \pm}^{\tilde{}}\left(s_{0}, z_{0}, w_{0}\right)= & \left\{(s, z, w) ;\left(\left|z-z_{0}\right|^{2}+\left|w-w_{0}\right|^{2}\right)^{1 / 2}\right. \\
& \left.\leqq M\left(s_{0}-s\right), s-s_{0} \leqq \pm\left(z-z_{0}\right) \leqq 0\right\}
\end{aligned}
$$

is a dependence domain of $\left(s_{0}, z_{0}, w_{0}\right)$ of the Cauchy problem for $P_{ \pm}$. Hence, the solution $u_{ \pm}^{\tilde{ \pm}}$ of $\left(\mathrm{C} . \mathrm{P}_{\circ}\right)_{ \pm}$satisfies $\tilde{u_{ \pm}}=0$ on $S_{ \pm}^{\tilde{*}}$. This means that $u_{ \pm}(t, x, y)=u_{ \pm}^{\sim}(t \pm x, x, y)=0$ on $S_{ \pm}$. Thus, we can solve (R. G. P.) ${ }_{ \pm}$. We can also prove the existence of good dependence domains from the fact that $\Gamma_{ \pm}^{\tilde{ \pm}}\left(s_{0}, z_{0}, w_{0}\right)$ is a dependence domain of $\left(s_{0}, z_{0}, w_{0}\right)$ of the Cauchy problem for $P_{ \pm}^{\sim}$.

\section{References}

[1] Hasegawa, Y., On the $C^{\infty}$-Goursat problem for equations with constant coefficients, J. Math. Kyoto Univ., 19 (1979), 125-151.

[2] Ivrii, V. Ya. and Petkov, V. M., Necessary conditions for the Cauchy problem for non-strictly hyperbolic equations to be well-posed, Russian Math. Surveys, 29 (1974), 1-70. (Uspekhi Mat. Nauk, 29 (1974), 3-70.)

[3] Ivrii, V. Ya., Sufficient conditions for regular and completely regular hyperbolicity, Trans. Moscow Math. Soc., 33 (1978), 1-65. (Trudy Moskov. Mat. Obšz., 33 (1976), 3-66.)

[4] Iwasaki, N., The Cauchy problem for effectively hyperbolic equations (a special case), J. Math. Kyoto Univ., 23 (1983), 503-562.

[5] Nishitani, T., On the $\mathscr{E}$-well-posedness for the Goursat problem with constant coefficients, J. Math. Kyoto Univ., 20 (1980), 179-190.

[6] - Une remarque sur le problème de Goursat, Séminaire sur les équations aux dérivées partielles hyperboliques et holomorphes (1981-82), J. Vaillant, Université de Paris II. 